\title{
Measurements of neonatal bilirubin and albumin concentrations: a need for improvement and quality control
}

\author{
Deirdre E. van Imhoff • Peter H. Dijk • \\ Cas W. Weykamp • Christa M. Cobbaert • \\ Christian V. Hulzebos • \\ On behalf of the BARTrial Study Group
}

Received: 4 October 2010 / Accepted: 14 December 2010 /Published online: 7 January 2011

(C) The Author(s) 2011. This article is published with open access at Springerlink.com

\begin{abstract}
Accurate and precise bilirubin and albumin measurements are essential for proper management of jaundiced neonates. Data hereon are lacking for Dutch laboratories. We aimed to determine variability of measurements of bilirubin and albumin concentrations typical for (preterm) neonates. Aqueous, human serum albumin-based samples with different concentrations of bilirubin $(100,200$, $300,400$, and $500 \mu \mathrm{mol} / \mathrm{L})$ and albumin $(0,10,15,20,25$, and $30 \mathrm{~g} / \mathrm{L}$ ) were sent to laboratories of all Dutch neonatal intensive care units $(n=10)$. Bilirubin and albumin recoveries of the specimens were measured using locally available routine analytical methods. The mean, standard deviation, and coefficients of variations (CV) were calculated per sample. Bilirubin concentrations were underestimated in the absence of albumin (maximal CV 26.0\%). When the albumin concentration was 10 or $20 \mathrm{~g} / \mathrm{L}$, the bilirubin concentrations of the samples were overestimated (maximal CV $14.1 \%$ and $9.2 \%$, respectively). Variability increased with higher weighed-in bilirubin concentrations. Measured albumin levels were $\sim 10 \%$ lower than albumin levels of manufactured samples. Bilirubin concentration did not influence albumin measurements. The maximal $\mathrm{CV}$ was $6.8 \%$. In conclusion, interlaboratory vari-
\end{abstract}

\footnotetext{
D. E. van Imhoff • P. H. Dijk $\cdot$ C. V. Hulzebos $(\bowtie) \cdot$

On behalf of the BARTrial Study Group

Division of Neonatology, Department of Pediatrics, Beatrix Children's Hospital, University Medical Center Groningen, Hanzeplein 1,

9700 RB Groningen, The Netherlands

e-mail: c.hulzebos@bkk.umcg.nl

C. W. Weykamp

Department of Clinical Chemistry, Queen Beatrix Hospital,

Winterswijk, The Netherlands

C. M. Cobbaert

Department of Clinical Chemistry,

University Medical Center Leiden,

Leiden, The Netherlands
}

ability of bilirubin and albumin measurements is high. Recalibration and introduction of a specific quality assessment scheme for neonatal samples is recommended to ensure exchangeability of bilirubin and albumin measurements among laboratories and to control the observed large variability.

Keywords Albumin · Bilirubin · Unconjugated hyperbilirubinemia $\cdot$ Neonates

\section{Introduction}

The risk of severe hyperbilirubinemia and bilirubin neurotoxicity in newborn infants underlines the need for accurate and precise bilirubin and albumin measurements as a part of management guidelines for hyperbilirubinemia. To prevent severe unconjugated hyperbilirubinemia and bilirubin neurotoxicity, (inter)national guidelines are used to standardize management of jaundiced neonates $[1,3,11,14]$. Treatment of unconjugated hyperbilirubinemia is based on the measurement of total serum bilirubin (TSB) concentration. Measurement of albumin concentration is recommended because a low albumin concentration is considered a risk factor for bilirubin neurotoxicity, resulting in lower TSB treatment thresholds or albumin infusion [1].

Variability of bilirubin and albumin measurements will obviously affect treatment decisions. In general, variability of routine chemical parameters is controlled by quality assessment schemes using external quality assessment (EQA) samples. In The Netherlands, human matrix-based, commutable EQA samples provided by the Dutch Foundation for Quality Assessment in Clinical Laboratories (in Dutch: SKML) are biweekly analyzed. However, bilirubin and albumin concentrations of these EQA samples typically represent adult ranges with a maximal bilirubin concentration of $80 \mu \mathrm{mol} / \mathrm{L}$ and a minimal albumin concentration of 
$30 \mathrm{~g} / \mathrm{L}$. Higher concentrations of bilirubin and lower concentrations of albumin are frequently encountered in (preterm) neonates. Data on variability of bilirubin and albumin measurements in the neonatal range are scarce and unavailable for Dutch laboratories [7]. In this study, we aimed to assess variability in measurements of bilirubin and albumin concentrations typical for neonates.

\section{Methods}

Participating laboratories

To analyze the variability of bilirubin and albumin measurements, the Dutch SKML distributed samples to laboratories of all $(n=10)$ Dutch Neonatal Intensive Care Units (NICUs). The NICUs were included because NICUs - and not general hospitals - are involved in the care of preterm infants of 32 or less weeks of gestation having an increased risk of developing hyperbilirubinemia and bilirubin neurotoxicity.

\section{Specimens}

The Dutch SKML manufactured artificial, aqueous, albumin-based EQA samples to evaluate bilirubin and albumin assay performance in the neonatal concentration range. In a Tris- $\mathrm{HCl}$ buffer $(\mathrm{pH}$ 7.4) with $9 \mathrm{~g} / \mathrm{L} \mathrm{NaCl}$, human serum albumin (HSA) (Sigma A 1653) was dissolved to achieve albumin concentrations ranging from 0 to $30 \mathrm{~g} / \mathrm{L}(0,10,15,20,25$, and $30 \mathrm{~g} / \mathrm{L})$. Unconjugated bilirubin (Sigma B4146) was dissolved (protected from light) and added to the albumin solutions immediately after complete solution. Bilirubin concentrations ranged from 100 to $500 \mu \mathrm{mol} / \mathrm{L}(100,200,300,400$, and $500 \mu \mathrm{mol} / \mathrm{L})$. Table 1 shows the combinations of bilirubin and albumin concentrations in the samples. After manufacture, the samples were frozen below $-70^{\circ} \mathrm{C}$, shipped on dry ice to the participating laboratories, and stored frozen until analysis. All EQA samples were analyzed in April 2008.

\section{Analytical devices}

In the clinical chemistry laboratories of the corresponding NICUs $(n=10)$, bilirubin and albumin recoveries of the processed EQA samples were evaluated using locally available routine analytical methods. Measurements on point of care analyzers were not included. All routine methods were standardized according to the instructions of the manufacturers. All laboratories participate in the regular EQA program of the SKML with maximal bilirubin concentrations of $80 \mu \mathrm{mol} / \mathrm{L}$ and minimal albumin concentrations of $30 \mathrm{~g} / \mathrm{L}$, enabling them to monitor and control albumin and bilirubin performance in the normal and slightly abnormal concentration ranges. The majority of the samples was measured in triplicate; a minority (one center) in duplicate.

\section{Statistical analysis}

The weighed-in concentrations of bilirubin and albumin in the processed EQA samples were compared with the measured concentrations as recovered by the clinical chemistry laboratory using routine methods. The mean, standard deviation (SD), range, and coefficients of variation (CV) were calculated per measured sample using Microsoft Office Excel (Microsoft Corporation, Redmond, WA, USA) and SPSS for Windows (version 16.0, Chicago, IL, USA).

\section{Results}

Analyzers and methods

Bilirubin and albumin measurements in the NICU laboratories were performed with locally available analytical methods, applied on routine clinical chemistry analyzers. Table 2 shows the analytical devices and corresponding methods used in the NICU laboratories.

\section{Bilirubin}

Panels a, b, and c of Fig. 1 show the measured versus the weighed-in bilirubin concentrations in samples with albumin concentrations of 0,10 , and $20 \mathrm{~g} / \mathrm{L}$, respectively. Underestimation of bilirubin concentrations occurred in the absence of albumin (Fig. 1a). When the albumin concentration was 10 or $20 \mathrm{~g} / \mathrm{L}$ (Fig. 1b, c), the bilirubin concentrations of the samples were overestimated. Independent of the albumin concentration in the sample, a large interlaboratory vari-

Table 1 Combinations of albumin and bilirubin concentrations in manufactured EQA samples

\begin{tabular}{lrrrrrrrrrrrrrrrrrrrrr}
\hline Sample & 1 & 2 & 3 & 4 & 5 & 6 & 7 & 8 & 9 & 10 & 11 & 12 & 13 & 14 & 15 & 16 & 17 & 18 & 19 & 20 \\
\hline Albumin $(\mathrm{g} / \mathrm{L})$ & 0 & 0 & 0 & 0 & 0 & 10 & 10 & 10 & 10 & 10 & 10 & 15 & 20 & 20 & 20 & 20 & 20 & 20 & 25 & 30 \\
Bilirubin $(\mu \mathrm{mol} / \mathrm{L})$ & 0 & 100 & 200 & 300 & 400 & 0 & 100 & 200 & 300 & 400 & 500 & 0 & 0 & 100 & 200 & 300 & 400 & 500 & 0 & 0 \\
\hline
\end{tabular}

$17.1 \mu \mathrm{mol} / \mathrm{L}=1 \mathrm{mg} / \mathrm{dL}$ bilirubin 
Table 2 Analytical devices and methods used in Dutch NICU laboratories

$D P D$ : 2,5-DiCl-phenyl

diazonium

${ }^{a}$ Unistad Salm and Kipp BV

${ }^{\mathrm{b}}$ Pfaff Technik \& Medizin
Devices

Method

Number of NICUs

Devices used for bilirubin measurement

Roche Modular

Reichert Unistat bilirubinometer $^{\mathrm{a}}$

Bilimeter $3^{\mathrm{b}}$

Beckman Coulter

Abbot Aeroset

Devices used for albumin measurement

Roche Modular

DPD

6

P800 module

Automatic

Beckman Coulter

Synchron Lx20

DxC 800

Abbott Aeroset
Spectrophotometric 1

Spectrophotometric 1

Jendrassik Grof 1

Jendrassik Grof 1

7

Bromcresol green 6

Bromcresol purple 1

2

Bromcresol green

Bromcresol purple

Bromcresol purple

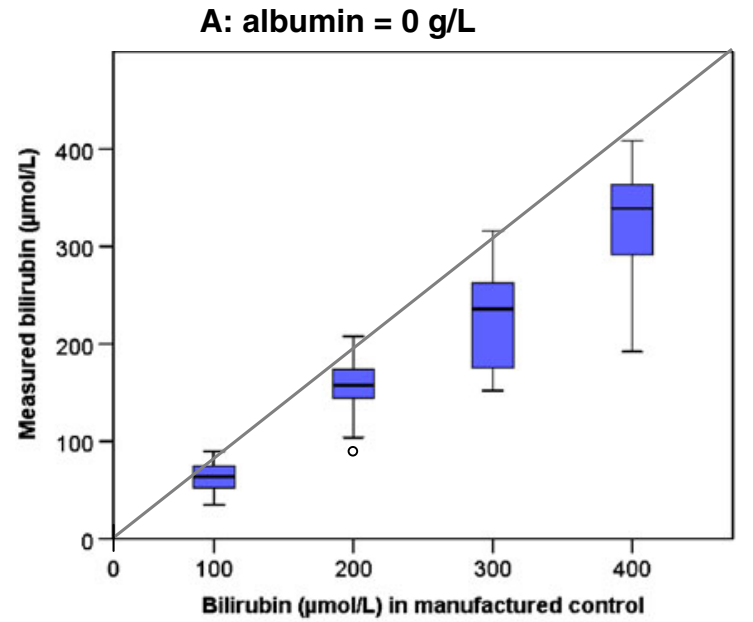

B: albumin $=10 \mathrm{~g} / \mathrm{L}$

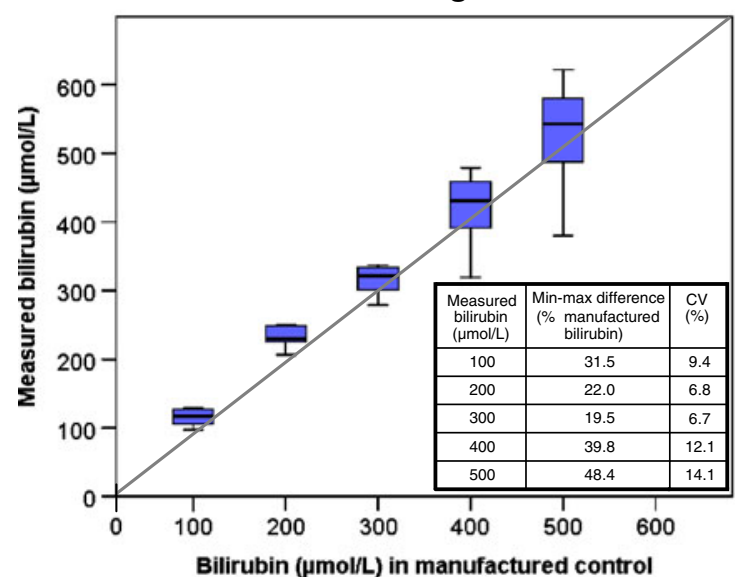

Fig. 1 a-c Variability of bilirubin measurements. Measured versus weighed-in bilirubin concentrations $(17.1 \mu \mathrm{mol} / \mathrm{L}=1 \mathrm{mg} / \mathrm{dL}$ bilirubin $)$ in manufactured samples with albumin concentrations of 0,10 , and $20 \mathrm{~g} / \mathrm{L}$, respectively. The median of measured bilirubin concentration is marked by the horizontal line in the central box. The boxes are limited by the 25 th and 75 th percentile. The whiskers represent

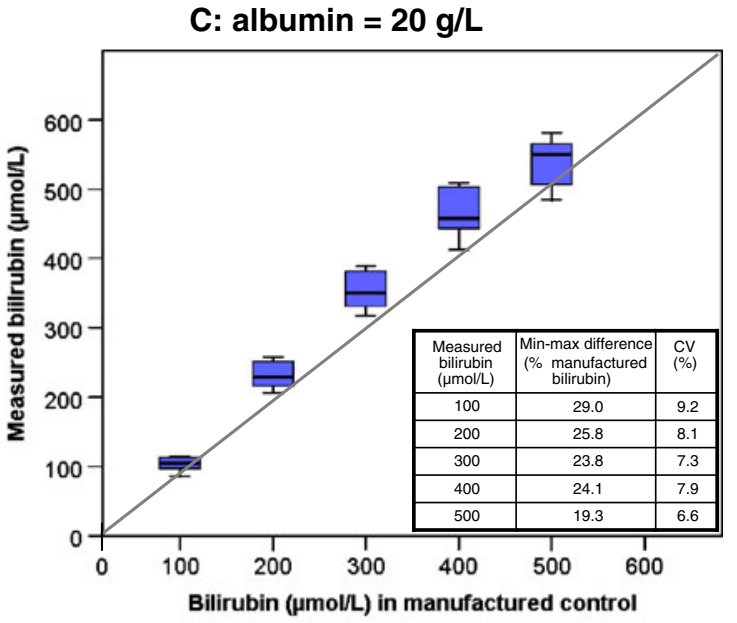

minimum and maximum measured bilirubin concentrations. Outliers (white circle) are depicted separately. Coefficients of variations (CV) are shown in Fig. $1 \mathbf{b}$ and $\mathbf{c}$ as well as the difference between the minimal and maximal measured bilirubin concentration, which is expressed as percentage of the weighed-in bilirubin concentration 
ability was found. The interlaboratory variability increased with increasing bilirubin concentrations (Fig. 1b, c). For example, for a bilirubin concentration of $100 \mu \mathrm{mol} / \mathrm{L}$ and an albumin concentration of $10 \mathrm{~g} / \mathrm{L}$, the range in measured bilirubin concentration was 97 to $128.5 \mu \mathrm{mol} / \mathrm{L}$ $(31.5 \mu \mathrm{mol} / \mathrm{L}), \sim 30 \%$ of the concentration in the sample (Fig. 1b). For bilirubin concentrations with an albumin concentration of $20 \mathrm{~g} / \mathrm{L}$, this increase in interlaboratory variability was less (Fig. 1c). Maximal interlaboratory coefficients of variation of measured bilirubin concentrations were $26.0 \%, 14.1 \%$, and $9.2 \%$ for samples with albumin concentrations of 0,10 , and $20 \mathrm{~g} / \mathrm{L}$, respectively.

\section{Albumin}

Table 3 shows the mean measured albumin concentrations of samples spiked with bilirubin ranging from 0 to $500 \mu \mathrm{mol} / \mathrm{L}$ (samples 6 to 20 in Table 1). Measured albumin concentrations were $\sim 10 \%$ lower than the concentrations of albumin dissolved in the samples. Maximal interlaboratory $\mathrm{CV}$ was $6.8 \%$. Unlike the albumin concentration in the bilirubin measurements, bilirubin concentration did not affect albumin measurements. For example, mean $( \pm \mathrm{SD})$ measured albumin concentration of the samples with an albumin concentration of $10 \mathrm{~g} / \mathrm{L}$ and six different bilirubin concentrations (samples 6 to 11 in Table 1) was $8.9( \pm 0.6) \mathrm{g} / \mathrm{L}$. For samples with an albumin concentration of $20 \mathrm{~g} / \mathrm{L}$ (samples 13 to 18 in Table 1), the mean $( \pm \mathrm{SD})$ albumin concentration was $18.0( \pm 0.7) \mathrm{g} / \mathrm{L}$.

\section{Discussion}

This study demonstrates 1) a large variability of neonatal bilirubin and albumin concentration measurements between

Table 3 Measured albumin concentrations

\begin{tabular}{lrc}
\hline Albumin $(\mathrm{g} / \mathrm{L})$ & Mean \pm SD (range) & CV (\%) \\
\hline 30 & $27.2 \pm 1.0(26.3-29.0)$ & 3.5 \\
25 & $22.6 \pm 0.9(21.5-24.3)$ & 4.0 \\
$20^{\mathrm{a}}$ & $18 \pm 0.7(17.3-19.2)$ & 3.7 \\
15 & $13.3 \pm 0.7(12.5-14.6)$ & 5.3 \\
$10^{\mathrm{a}}$ & $8.9 \pm 0.6(8.1-10.0)$ & 6.8 \\
\hline
\end{tabular}

Data represent the results of ten laboratories for the measured concentrations of EQA samples 6 to 20 shown in Table 1 with albumin concentrations ranging from 10 to $30 \mathrm{~g} / \mathrm{L}$. Results are expressed as mean $\pm \mathrm{SD}$ (ranges) with interlaboratory coefficients of variation $(\mathrm{CV})$ per concentration (percentage)

${ }^{a}$ Albumin concentrations of 10 and $20 \mathrm{~g} / \mathrm{L}$ describe mean albumin concentrations of samples with six different bilirubin concentrations laboratories of Dutch NICUs potentially affecting treatment of jaundiced newborn infants and 2) the lack of standardized devices/methodology for neonatal bilirubin and albumin measurements on laboratories of Dutch NICUs. Differences in applied laboratory methodology can, theoretically, result in interlaboratory variability. Discrepancies up to $13 \%$ of bilirubin measurements have been observed in the past between wet chemistry and dry chemistry analyzers using quality control samples [2]. Even greater differences of 30\% higher results for wet versus dry bilirubin measurements in human serum samples have been demonstrated [2]. In our study, all laboratories used wet chemistry analyzers so the observed and rather large interlaboratory variability is not due to differences in wet versus dry chemistry methodology. Our results are in agreement with data of Vreman et al., who reported similar interlaboratory variability of bilirubin measurements, applying similar methodology and/or devices [15].

Interlaboratory variability can be traced, at least in part, to the height of the bilirubin concentration and to the use of different matrixes (i.e., bovine versus human serum based), as has been addressed previously [5-7, 13]. In 1982, Schreiner et al. studied the bilirubin variability of stabilized liquid quality control sera spiked with different concentrations of bilirubin. An interlaboratory variability comparable with our findings was found as well as an increased variability in the higher bilirubin concentrations [13]. Matrix effects refer to the difference in behavior of patient samples versus processed (EQA) samples. In bilirubin analysis, the protein content may affect the molar absorptivity of the bilirubin reaction. Frequently used matrices for preparing calibrators are human serum and human or bovine serum albumin-based solutions. The variable and unpredictable underestimation of conjugated bilirubin in bovine serum renders the relationship between the actual bilirubin content and assigned values in calibrators unreliable and dependent on the quality and source of the bovine products. There is no reliable method for accurately measuring the concentration of bilirubin in calibrators and controls in bovine sera from commercial sources. The use of these products compromises the accuracy of bilirubin measurements in newborn infants. Therefore, human instead of bovine serum should be used for preparing bilirubin calibrators to minimize variability. In 2003, the College of American Pathologists included in the Neonatal Bilirubin Surveys a human serum-based sample enriched solely with unconjugated bilirubin [6,7]. Native human serum is the most commutable matrix among different laboratory methods because it mimics the entire serum composition [6]. Yet, the use of the same matrix does not necessarily imply minimal variability. Recently, the Dutch Foundation for Quality Assessment in Clinical Laboratories initiated a study with two native human serum samples 
spiked with two different total bilirubin concentrations (30 and $70 \mu \mathrm{mol} / \mathrm{L}$ ) to assess standardization of bilirubin measurements in The Netherlands among different manufacturers. In analogy to our results, a large interlaboratory variability was demonstrated [4].

Apart from the large interlaboratory variability, underestimation of the bilirubin concentration was observed in albumin-free samples. This underestimation of bilirubin concentration in the albumin-free samples is very likely a pre-analytical problem: albumin is essential to dissolve and stabilize bilirubin.

This study illustrates significant interlaboratory differences among bilirubin measurements using aqueous HSA-based samples. Yet, one should be careful with extrapolation of these findings to native neonatal samples as aqueous HSA-based samples behave differently. Moreover, the variability was deduced from the weighed-in calculated bilirubin and albumin concentration and not by value assignment using an internationally recognized reference method. Notwithstanding these limitations, a large interlaboratory variability of bilirubin and albumin measurements is noticed.

International management guidelines of infants with unconjugated hyperbilirubinemia are based on total serum bilirubin (TSB). However, bilirubin neurotoxicity may be better predicted by the unbound or free bilirubin, i.e., the fraction of bilirubin not bound to plasma proteins (mainly albumin), than by TSB. Unfortunately, free bilirubin measurements are laborious and not routinely available, in contrast to TSB measurements [16]. Inaccurate and/or imprecise bilirubin measurements can potentially result in overtreatment or undertreatment of jaundiced neonates, including inappropriate blood sampling. Overtreatment exposes the newborn infants to unnecessary risks. Phototherapy, although generally considered safe, may result in retinal damage (when eyes are unprotected from phototherapylight), skin pathology, and an increased insensible water loss [9]. Exchange transfusions are invasive and associated with considerable morbidity and mortality (estimated to occur in 50 and 3 per 1,000 procedures, respectively) $[1,10]$. In contrast, underestimation of bilirubin levels may result in undertreatment, possibly contributing to a reemergence of bilirubin neurotoxicity in jaundiced infants [8].

Variability of bilirubin and albumin measurements will for obvious reasons negatively influence standardizing care for jaundiced newborn infants. Standardized care based on the best available evidence has been demonstrated to improve pediatric and adult patient outcomes [12]. To standardize the management of unconjugated hyperbilirubinemia, accurate and precise measurements of bilirubin and albumin concentrations are inevitably. Monitoring laboratory performance is of key importance herein as has also been internationally recognized [7].
Recommendations to improve and standardize care of jaundiced newborn infants are, according to the authors of this study, twofold. First, accuracy of bilirubin and albumin measurements needs to be improved and the desired quality specifications should be defined. In agreement with international experts, we recommend a total error for neonatal bilirubin of maximal $10 \%$ of the reference method [7]. Interestingly, two manufacturers advocated to recalibrate their bilirubin assays for measurement of total bilirubin in the last quarter of 2008 . A downscaling of formerly assigned concentrations to calibrators of $-11 \%$ to $-20 \%$ was recommended [4]. Second, commutable external quality control samples with high neonatal bilirubin levels should be used to monitor the required reduction in interlaboratory variability. Although originally recommended in the twentieth century, this recommendation is currently still valid for many countries [15]. In The Netherlands, in addition to "adult range" bilirubin and albumin samples, bilirubin and albumin concentrations typically encountered in jaundiced neonates, i.e., $200-500 \mu \mathrm{mol} / \mathrm{L}$ and $15-25 \mathrm{~g} / \mathrm{L}$, respectively, are nowadays biweekly monitored by clinical chemistry laboratories involved in the care for neonates with unconjugated hyperbilirubinemia.

\section{Conclusions}

To comply with international guidelines for the management of neonates with unconjugated hyperbilirubinemia, exchangeability of bilirubin and albumin measurements among laboratories is essential. Our current findings, although in aqueous HSA samples, apparently point to unacceptably high (interlaboratory) variability of bilirubin and albumin measurements. To analyze and improve the interlaboratory variability, a tailor-made quality assessment scheme for neonatal samples (human serum based) is available in The Netherlands since January 2010.

\section{Acknowledgments}

BARTrial Study Group Academic Medical Center University of Amsterdam-Mrs. L. van Toledo-Eppinga, MD, PhD*; J. Fischer, $\mathrm{PhD}^{* *}$. University Medical Center Maastricht-A.L.M. Mulder, MD, $\mathrm{PhD}^{*}$; O. Bekers, PhD**. Erasmus Medical Center Rotterdam-P. Govaert, MD, PhD*; R. de Jonge, PhD**. Isala Clinics Zwolle-R.A. van Lingen, $\mathrm{MD}, \mathrm{PhD}^{*}$; R.J. Slingerland, $\mathrm{PhD}^{* *}$. University Medical Center Leiden-E. Lopriore, MD, PhD*; Mrs. M.C. Cobbaert, $\mathrm{PhD}^{* *}$. Maxima Medical Center Veldhoven-J. Buijs, MD*; Prof. H.L. Vader, $\mathrm{PhD}^{* *}$. University Medical Center Groningen-Mrs. D.E. van Imhoff, MD; P.H. Dijk, MD, PhD*; C.V. Hulzebos, MD, PhD*; L.J. van Pelt, MD, PhD**. University Medical Center St. Radboud Nijmegen-K.D. Liem, MD, PhD*; M.A.M.A. Roelofs-Thijssen, PhD**. University Medical Center Utrecht-Mrs. M.J.N.L. Benders, $\mathrm{MD}, \mathrm{PhD}^{*} ; \mathrm{H}$. Kemperman, $\mathrm{PhD}^{* *}$. University Medical Center Amsterdam-Prof. W.P.F. Fetter, MD, PhD*; A.A. Bouman, PhD**. *Pediatrician-neonatologist; **clinical chemist 


\section{Conflict of interest None.}

Open Access This article is distributed under the terms of the Creative Commons Attribution Noncommercial License which permits any noncommercial use, distribution, and reproduction in any medium, provided the original author(s) and source are credited.

\section{References}

1. American Academy of Pediatrics (2004) Management of hyperbilirubinemia in the newborn infant 35 or more weeks of gestation. Pediatrics 114:297-316

2. Apperloo JJ, van der GraafF, Scharnhorst Vet al (2005) Do we measure bilirubin correctly anno 2005? Clin Chem Lab Med 43:531-535

3. Bhutani VK, Maisels MJ, Stark AR et al (2008) Management of jaundice and prevention of severe neonatal hyperbilirubinemia in infants $>$ or $=35$ weeks gestation. Neonatology 94:63-67

4. Cobbaert C, Weykamp C, Hulzebos CV (2010) Bilirubin standardization in the Netherlands: alignment within and between manufacturers. Clin Chem 56(5):872-873

5. Doumas BT, Eckfeldt JH (1996) Errors in measurement of total bilirubin: a perennial problem. Clin Chem 42:845-848

6. Lo SF, Doumas BT, Ashwood ER (2004) Performance of bilirubin determinations in US laboratories — revisited. Clin Chem 50:190-194
7. Lo SF, Jendrzejczak B, Doumas BT (2008) Laboratory performance in neonatal bilirubin testing using commutable specimens: a progress report on a College of American Pathologists study. Arch Pathol Lab Med 132:1781-1785

8. Maisels MJ (2009) Neonatal hyperbilirubinemia and kernicterus - not gone but sometimes forgotten. Early Hum Dev 85 (11):727-732

9. Maisels MJ, McDonagh AF (2008) Phototherapy for neonatal jaundice. N Engl J Med 358:920-928

10. Maisels MJ, Watchko JF (2003) Treatment of jaundice in low birthweight infants. Arch Dis Child Fetal Neonatal Ed 88(6): F459-F463

11. Maisels MJ, Bhutani VK, Bogen D et al (2009) Hyperbilirubinemia in the newborn infant $>$ or $=35$ weeks' gestation: an update with clarifications. Pediatrics 124:1193-1198

12. Muething SE (2005) Improving patient outcomes by standardizing care. J Pediatr 147:568-570

13. Schreiner RL, Glick MR (1982) Interlaboratory bilirubin variability. Pediatrics 69:277-281

14. Van Imhoff DE, Dijk PH, Hulzebos CV (2009) Uniform intervention criteria for jaundice in hyperbilirubinemia in preterm infants. Ned Tijdschr Geneeskd 153:A94

15. Vreman HJ, Verter J, Oh W et al (1996) Interlaboratory variability of bilirubin measurements. Clin Chem 42:869-873

16. Wennberg R, Ahlfors C, Bhutani V et al (2006) Toward understanding kernicterus: a challenge to improve the management of jaundiced newborns. Pediatrics 117:474-485 